\title{
A STUDY OF MASS LOSS AND DUST FORMATION NEAR HOT STARS
}

\author{
K.S. Kuratov ${ }^{1,2}$, A.S. Miroshnichenko ${ }^{1,3}$, A.V. Kusakin ${ }^{4}$, N.Sh. Alimgazinova ${ }^{1,2}$, \\ A.Zh. Nuryzbaeva ${ }^{1,2}$, A.B. Manapbaeva ${ }^{1,2}$, A.K. Kuratova ${ }^{1,2}$ \\ ${ }^{1}$ National center of space exploration and technologies, Almaty, Kazakhstan, \\ kenes.kuratov@.mail.ru \\ ${ }^{2}$ Faculty of Physics and Technology, Al-Farabi Kazakh National University, \\ Almaty, Kazakhstan \\ ${ }^{3}$ Department of Physics and Astronomy, University of North Carolina \\ Greensboro, NC 27402-6170, USA, a_mirosh@uncg.edu \\ ${ }^{4}$ Fessenkov Astrophysical Institute, Observatory 23, Almaty, Kazakhstan
}

ABSTRACT. At present dust formation is well studied only near cool stars, whose surface temperatures are close to those of dust sublimation. Hot stars need to supply large amounts of circumstellar material to allow dust formation around them. Such conditions naturally exist near supergiants with masses over 25 $\mathrm{M}_{\odot}$. The theory of stellar evolution predicts that less massive stars do not provide enough matter for dust formation. Nevertheless, dust exists near dwarfs with the $\mathrm{B}[\mathrm{e}]$ phenomenon and giants of $\mathrm{A}-\mathrm{G}$ spectral types which do not belong to star formation regions.

A large group of objects with the $\mathrm{B}[\mathrm{e}]$ phenomenon with extremely strong emission-line spectra that are neither young nor highly evolved has been recently identified. They are called FSCMa type objects. Their infrared excesses imply a large amount of recently created dust. Therefore, these objects can noticeably contribute to the Galactic dust content, but they have not been taken into consideration from this perspective.

Key words: - Stars: emission-line, Be - Stars: evolution - (Stars:) circumstellar matter - (Stars:) binaries: general

\section{Introduction}

The $\mathrm{B}[\mathrm{e}]$ phenomenon is defined as the presence of forbidden emission lines and strong infrared excess due to radiation from circumstellar dust in the spectra of B-type stars. It was discovered nearly 40 years ago in 65 Galactic objects by Allen \& Swings (1976). The phenomenon exists at different evolutionary stages of several stellar groups: pre-main-sequence Herbig Ae/Be stars, compact Planetary Nebulae, symbiotic binary systems, and some massive supergiants (Lamers et al., 1998). However, the nature of 30 objects out of these 65 was not determined, and they were called unclassified objects with the $\mathrm{B}[\mathrm{e}]$ phenomenon.

Miroshnichenko (2007) analyzed properties of the unclassified objects and summarized them as follows:

1. early-B to early-A type optical continuum with strong emission lines of hydrogen, Fe II, [O I], and sometimes of [Fe II] and [O III] (absorption lines from the hot star atmosphere may be present as well, but they are frequently veiled by the circumstellar continuum);

2. a large IR excess that peaks at $\lambda \sim 10-30 \mu \mathrm{m}$ and sharply decreases toward longer wavelengths;

3. location outside of star-forming regions; and

4. a secondary companion (so far discovered in about one third of the group members) which can be a fainter and cooler normal star or a degenerate object.

The group was named FS CMa type objects using the variable star name of the most typical object with the $\mathrm{B}[\mathrm{e}]$ phenomenon (FS CMa $=\mathrm{HD} 45677$, Swings 2006). Miroshnichenko et al. (2007) expanded the group with 10 objects found in the IRAS catalog by cross-identification with catalogs of optical positions. Miroshnichenko et al. (2011) found 20 more group objects by cross-correlating the Hamburg catalog of emission-line stars (Kohoutek \& Wehmeyer, 1999), IRAS (IPAC, 1986), and 2MASS (Cutri et al., 2003) catalogs. Property 2 listed above is crucial to separate FS CMa objects from other hot emission-line stars. This criterion was applied to objects detected by the IRAS satellite, which has had a low sensitivity 
and reliably measured fluxes only from the brightest objects. Other photometric criteria to find more candidates to the FS CMa group are presented below.

\section{Properties of the FS CMa group objects}

One of the main problems with determining the nature and evolutionary state of the FS CMa objects are transition of absorption lines, which form in the atmosphere of the B-type star and serve as diagnostics for the star temperature and luminosity, into emission caused by the circumstellar matter. This matter also produces continuum emission that fills in or weakens the absorption lines. Such a situation hampers luminosity and distance determination, especially in the absence of high-quality data. This was the case for many relatively faint objects (visual brightness $V \geq 10 \mathrm{mag}$ ) in 1980's and 1990's.

Another problem is a combination of features of stars of different masses or evolutionary stages in one object. For example, such a combination can be a strong IR excess, typical of pre-main-sequence stars, and an extremely strong emission-line spectrum, typical of evolved high-luminosity stars (supergiants).

We began systematic medium- and high-resolution spectroscopic and multicolor photometric observations of the group objects in the end of 1990's. Even single high-resolution spectra that we obtained for some newly found objects for the first time (e.g., Miroshnichenko et al., 2000) allowed us to estimate distances to them using kinematical (radial velocity) information and interstellar extinction laws in their directions. Effective temperatures of the underlying Btypes stars were estimated from excitation of the emission lines and dereddned optical photometry. As a result, we placed a number of FSCMa objects in the Hertzsprung-Russell diagram (HRD, see Fig. 1 where positions of supergiants with the $\mathrm{B}[\mathrm{e}]$ phenomenon is shown for comparison) and showed that they were not high-luminosity or high-mass stars.

FS CMa objects show very strong emission-line spectra, much stronger (Miroshnichenko, 2008) than those of other groups of objects in the same photospheric temperature range $(10000-30000 \mathrm{~K})$. This suggests a large amount of circumstellar gas around the stars. Theory of stellar evolution cannot explain such large amounts by mass loss from a single star of masses predicted by positions of FS CMa objects in HRD. This indirectly suggests that FS CMa objects can be binary systems, in which a strong mass transfer between the stellar components is responsible for both circumstellar gas and dust.

Another distinct feature of FS CMa objects is a fast decrease of their IR flux, which is caused by radiation of the circumstellar dust, towards longer wavelengths starting from a peak at $\lambda \sim 10-30 \mu \mathrm{m}$ (see

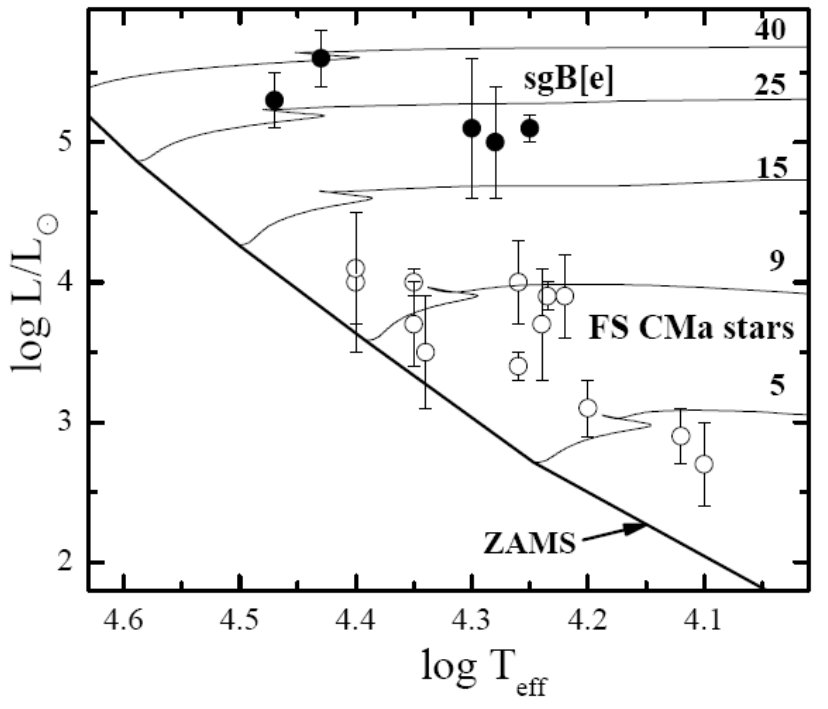

Figure 1: A Hertzsprung-Russell diagram for Galactic FS CMa stars and B[e] supergiants (B[e]sg) with known parameters (from Miroshnichenko 2007). Solid lines: the Zero-Age Main-Sequence (ZAMS) and evolutionary tracks for single rotating stars (Ekström et al. 2012) with initial masses in solar units indicated.

Fig. 2). Their IR color-indices are found in the same region of the IRAS color-color diagram as those of much cooler stars, which do not produce enough UV radiation to heat distant colder dust that radiates in the far-IR. This feature can be explained by compactness of the dust distribution near FS CMa objects and distinguishes them from pre-main-sequence Herbig Ae/Be stars, which have larger dusty disks and exhibit more far-IR radiation. Such an IR spectral energy distribution is also different from those of young Planetary Nebulae, whose envelopes contain much colder dust that produces mostly far-IR excess radiation and little or no near-IR excess.

Based on the described properties, we propose a model of FS CMa objects that includes a binary system, where mass transfer between the two stars led to the circumstellar envelope/disk formation. The gas responsible for emission lines is mostly located around the hot star, while the dust exists in the circumbinary area. So far only 12 objects from the FS CMa group have been recognized as binaries. This is due to weakness of the secondary components contribution to the total spectrum of the system. Typically the secondary components are 2-4 magnitudes fainter than the Btype primaries in the optical region of spectrum (e.g., Miroshnichenko, 2007; Miroshnichenko et al., 2015).

The HRD location of FS CMa objects imply intermediate masses $\left(3-20 \mathrm{M}_{\odot}\right)$ for the hot primary components, while the secondary components are probably less massive. This suggests that the number of such systems in the Galaxy can be large. Since they pro- 


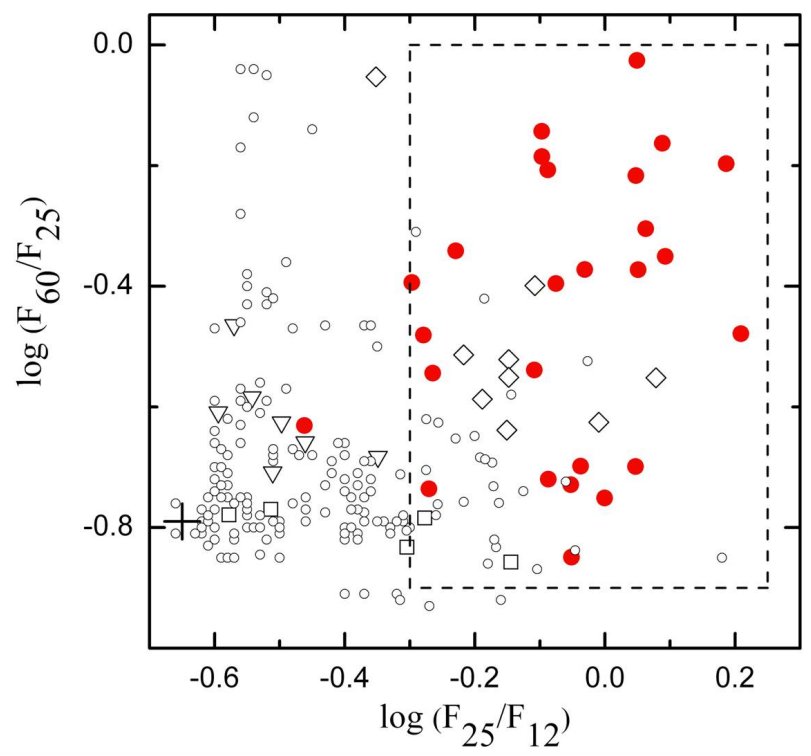

Figure 2: An IRAS color-color diagram for dusty objects. Symbols: FS CMa stars - red filled circles; symbiotic binaries - squares; carbon stars - triangles; AGB stars - circles; RV Tau stars (low-mass post-AGB objects) - diamonds. The dash-lined box shows location of the FS CMa stars. The large cross marks the stellar photospheric locus for all temperatures. $\mathrm{F}_{12}, \mathrm{~F}_{25}$, and $\mathrm{F}_{60}$ are IRAS fluxes at the corresponding wavelengths.

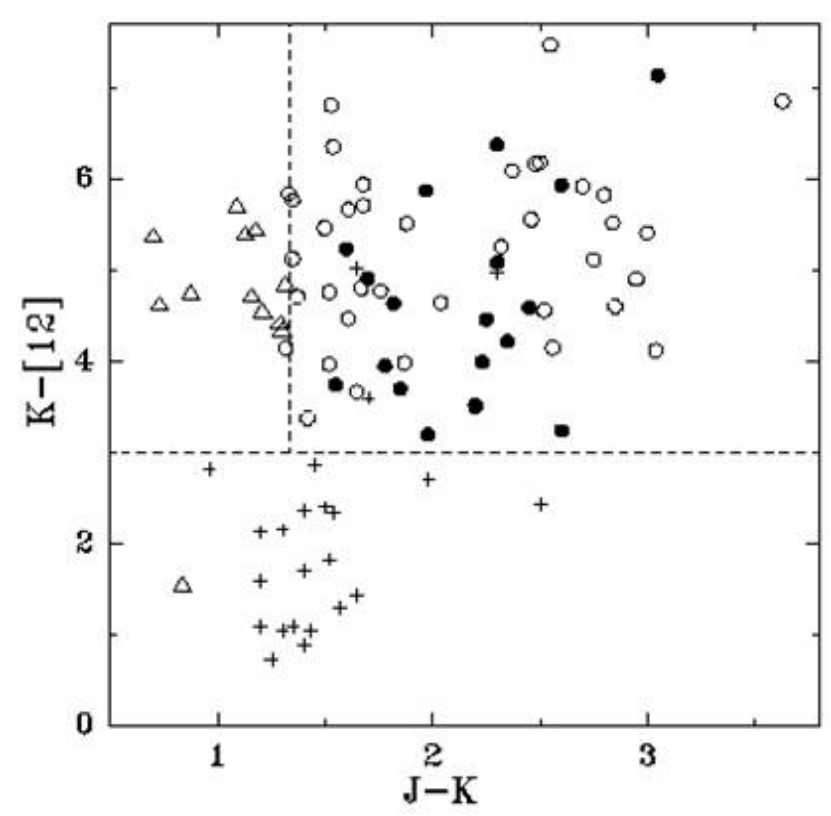

Figure 3: A photometric criterion that separates RV Tau stars (triangles), most cool stars (crosses), and FS CMa objects (groups members - filled circles, candidates to the group - open circles). [12] is the IRAS magnitude converted from the flux in the $12-\mu \mathrm{m}$ photometric band.

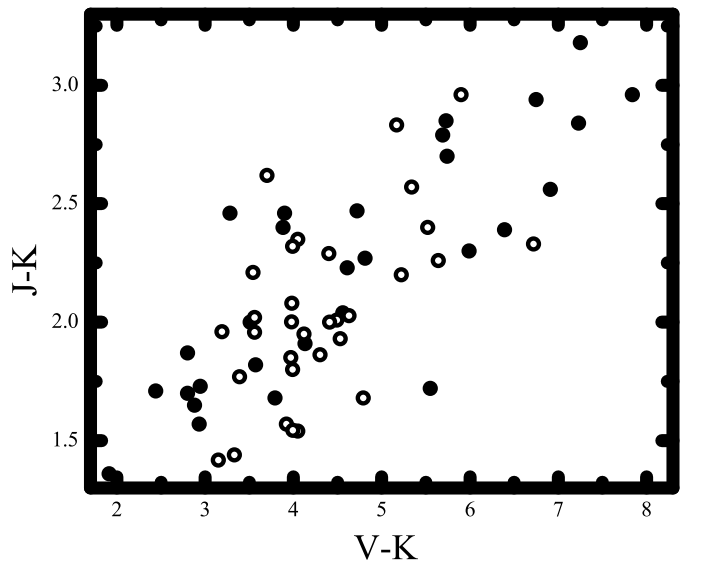

Figure 4: Optical-to-near-IR colors of the FS CMa group members and candidates. Symbols are the same as on the left panel.

duce dust, they can be important in the Galactic dusty budget. Therefore one could expect to find other such systems in the existing catalogs of photometric data or emission-line stars.

As photometric catalogs contain many more objects that spectroscopic ones and the IR spectral energy distribution can be used to separate FS CMa objects, we first looked in the IRAS catalog for new candidates to the group. However, the sensitivity of the IRAS photometric survey was low and allowed detection of only the brightest objects and those with the most dust. To expand the search opportunities, we analyzed other color-indices, including those in the near-IR and even the optical range. The goal was to compare the color-indices of FS CMa objects with those of other groups of objects with circumstellar dust. The results are shown in Figures 3 and 4.

\section{Results}

We collected optical and IR color-indices for representative samples of various objects showing the presence of circumstellar dust (e.g., pre-main-sequence Herbig Ae/Be stars, main-sequence Vega-type stars with debris dusty disks, Planetary Nebulae, symbiotic binaries, etc.) and proposed several criteria to search for FS CMa object candidates.

These criteria include the following:

1. $\mathrm{m}_{\mathrm{B}}-\mathrm{m}_{\mathrm{V}} \leq 1$ mag: separates out most cool stars but misses very reddened objects

2. $\mathrm{m}_{\mathrm{V}}-K \geq 2$ mag: separates out objects with no dust 
3. $J-K \geq 1.4$ mag: separates out low-mass postAGB objects

4. $K-[12] \geq 3$ mag: separates out most cool stars with no dust

In this list of criteria, $m_{B}$ and $m_{V}$ are the blue and visual magnitudes listed in the NOMAD catalog (Zacharias et al., 2004) are used, $J$ and $K$ are near-IR magnitudes at 1.25 and $2.2 \mu \mathrm{m}$, respectively; and [12] is the IRAS flux in $12-\mu \mathrm{m}$ band converted into magnitudes.

The NOMAD catalog contains photometric data that includes 3 optical bands (not exactly coinciding with the standard $B V R$ bands) and 3 near-IR bands from the 2MASS catalog. We started searching the entire sky for new candidates to the FS CMa group using the above criteria in NOMAD. This search is complemented by collecting information from other sources, such as IR surveys WISE (Wright et al., 2010) and AKARI (Murakami et al., 2007), and optical variability monitoring programs, such as ASAS (Pojmanski, 1997).

\section{Conclusions}

1. We found a new group of objects which create circumstellar dust and may be important for the dust production in the Galaxy. Currently the group has about 70 members and candidates. Most of them are fainter than $V \sim 11$ mag. The group is called the FS CMa type objects.

2. A current explanation for the group nature involves intermediate-mass binary systems at or after a stage of significant mass transfer due to a Roche lobe overflow by one of the stars in the system. This model is supported by calculations of binary evolution (van Rensbergen et al., 2008).

3. The FS CMa objects exhibit strong emission-line spectra indicative of a significant amount of circumstellar material in the system consistent with the assumption of mass transfer.

4. The secondary companion to the hot primary is typically a few times fainter that makes hard detecting them.

5. We continue a search for more group candidates, which will be identified using photometric criteria developed by us and observed spectroscopically to confirm the presence of the $\mathrm{B}[\mathrm{e}]$ phenomenon. So far we have searched about one half of the sky and found over 100 candidates. Our previous experience shows that most candidates found using our criteria show the presence of the $\mathrm{B}[\mathrm{e}]$ phenomenon.

\section{References}

Allen D.A. \& Swings J.-P.: 1976, A\&A, 47, 293.

Cutri R.M., Skrutskie M.F., Van Dyk S. et al.: 2003, CDS/ADC Collection of Electronic Catalogues, 2246 .

Ekström S., Georgy C., Eggenberger P. et al.: 2012, $A \& A, \mathbf{5 3 7}, \mathrm{A} 146$.

Kohoutek L. \& Wehmeyer R.: 1999, A\&AS, 134, 255.

IPAC: 1986, IRAS catalogue of Point Sources, Version 2.0, Joint IRAS Science W.G. (Vizier Catalog II/125).

Lamers H.J.G.L.M., Zickgraf F.-J., de Winter D., Houziaux L., Zorec J.: 1998, A\&A A, 340, 117.

Miroshnichenko A.S.: 2007, ApJ, 667, 497.

Miroshnichenko A.S.: 2008, in "Mass Loss from Stars and the Evolution of Stellar Clusters", eds. A. de Koter, L.Smith, and L.B.F.M.Waters, ASP Conf. Ser., 388, 205.

Miroshnichenko A.S., Chentsov E.L., Klochkova V.G. et al.: 2000, $A \mathscr{E} A S, \mathbf{1 4 7}, 5$.

Miroshnichenko A.S., Manset N., Kusakin A.V. et al.: 2007, ApJ, 671, 828.

Miroshnichenko A.S., Manset N., Polcaro V.F., Rossi C., Zharikov S.V.: 2011, In "Active OB stars: structure, evolution, mass loss and critical limits", Proc. of the IAU Symposium, 272, 260.

Miroshnichenko A.S., Zharikov S.V., Danford S., Manset N., Korčáková D., Kříček R., Šlechta M., Omarov Ch.T., Kusakin A.V., Kuratov K.S., Grankin K.N.: 2015, ApJ, 809, 129.

Murakami H., Baba H., Barthel P. et al.: 2007, PASJ, $\mathbf{5 9}, 369$.

Pojmanski G.: 1997, Acta Astronomica, 47, 467.

Swings J.-P.: 2006, In "Stars with the B[e] Phenomenon, eds. M.Kraus and A.S.Miroshnichenko, ASP Conf. Ser., 355, 3.

van Rensbergen W., De Greve J.P., De Loore C., Mennekens N.: 2008, A\&A, 487, 1129.

Wright E.L., Eisenhardt P.R.M., Mainzer A.K. et al.: 2010, AJ, 140, 1868.

Zacharias N., Monet D.G., Levine S.E., Urban S.E., Gaume R., Wycoff G.L.: 2004, Bull. American Astron.Soc., 36, 1418, (Vizier Catalog I/297). 